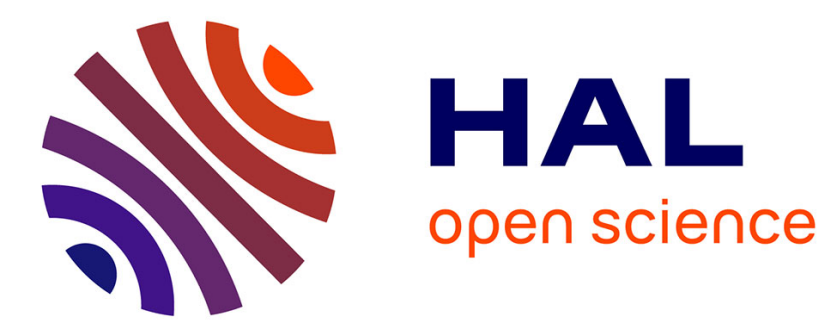

\title{
Weak-field magnetoresistance coefficients and the related magnetoresistance skewness effect
}

D.S. Kyriakos, O.E. Valassiades, N.A. Economou

\section{To cite this version:}

D.S. Kyriakos, O.E. Valassiades, N.A. Economou. Weak-field magnetoresistance coefficients and the related magnetoresistance skewness effect. Revue de Physique Appliquée, 1982, 17 (2), pp.49-54. 10.1051/rphysap:0198200170204900 . jpa-00244971

\section{HAL Id: jpa-00244971 https://hal.science/jpa-00244971}

Submitted on 1 Jan 1982

HAL is a multi-disciplinary open access archive for the deposit and dissemination of scientific research documents, whether they are published or not. The documents may come from teaching and research institutions in France or abroad, or from public or private research centers.
L'archive ouverte pluridisciplinaire HAL, est destinée au dépôt et à la diffusion de documents scientifiques de niveau recherche, publiés ou non, émanant des établissements d'enseignement et de recherche français ou étrangers, des laboratoires publics ou privés. 


\title{
Weak-field magnetoresistance coefficients and the related magnetoresistance skewness effect
}

\author{
D. S. Kyriakos, O. E. Valassiades and N. A. Economou \\ Department of Physics, Aristotelion University of Thessaloniki, Greece
}

(Reçu le 9 juin 1981, révisé le 7 octobre 1981, accepté le 27 octobre 1981)

\begin{abstract}
Résumé. - Ce travail présente une vue d'ensemble des mesures concernant les coefficients galvanomagnétiques dans le cas du champ faible afin de préciser la signification physique des résultats. La méthode est basée sur l'anisotropie induite dans le comportement électrique à cause du champ magnétique. L'accent est mis aussi sur l'explication de l'effet de magnétorésistance oblique. Une comparaison avec la méthode classique est présentée pour indiquer les avantages de notre méthode. Celle-ci est appliquée à des mesures sur Ge de type n pour lequel le modèle des bandes d'énergie permet de déduire le quotient $m_{1} / m_{t}$; ce quotient est utilisé pour obtenir le facteur de scattering, la densité et la mobilité des porteurs. Les résultats obtenus sont en accord avec les résultats antérieurs.
\end{abstract}

\begin{abstract}
This work presents an overall view of the measurements concerning the galvanomagnetic coefficients in the weak-field case with an attempt to emphasize the physical significance of the results. The method is based on the anisotropy induced in the electrical behaviour by the magnetic field. Emphasis is also given in explaining the magnetoresistance skewness effect. A comparison with the classical method is also presented to indicate the advantage of the method. Finally the method is analyzed for measurements on n-type Ge for which the energy band model is derived to obtain the $m_{1} / m_{t}$ ratio which in return is used to obtain the scattering factor, the carrier density and the drift mobility. The results are in accordance with previous findings.
\end{abstract}

1. Introduction. - This work presents an overall view concerning the methodology of the measurements of the galvanomagnetic coefficients (GMC) in the weak-field case presented in a series of previous papers [1-4] with an attempt to emphasize the advantage of the method and to link it with the physical significance of the results. The measurements are made basicaly by the Wasscher's method [5] which leads to the polar distribution of the resistivity on the sample plane. Thus the determination of the GMC is considerably simplified and a new effect is risen, the magnetoresistance skewness (MRS) effect. This effect is expected by the weak-field phenomenological theory. Unfortumately until now the MRS effect and its importance remained indefinite due to the 'in-use experimental configuration which allows magnetoresistance measurements only in one direction e.g. the direction of the sample rod.

Reference [4] is an experimental work on n-type Ge. In that work the MRS effect was established experimentally in accordance with the theory, which is based on the equation (1) [see the next paragraph].

Here, since we believe that the Wasscher's method presents some advantages over the classical experimental method we shall make a comparison of the two methods and of the results of the reference [4] with experimental results obtained by the classical configuration.

\section{A brief review of the traditional electric measu-} rements. - In the galvanomagnetic measurements one of the main problems is the determination of the weak-field magnetoresistance (WFMR) coefficients because they are linked to the various kinds of anisotropies in the band structure or of the scattering mechanisms. The WFMR coefficient $\rho_{i j k l}$ are connected in a direct way with the magnetoresistance effect as it is expressed by the well known relation

$$
E_{i}=\rho_{i j} J_{j}+\rho_{i j k} J_{j} B_{k}+\rho_{i j k l} J_{j} B_{k} B_{l}
$$

where $\bar{E}$ is the electric field intensity, $\bar{J}$ the current density and $\bar{B}$ the magnetic field induction. The other tensor elements $\rho$ have the usual meaning while the implied summation is carried over all possible values of all repeated indices.

The existence of the $\rho_{i j k l}$ coefficients shows that even in the case of isotropic materials the influence of the magnetic field has as a result an anisotropy in the electric resistivity.

There is a limit in the number of the distinct WFMR coefficients which can be determined by one sample, 
due to the sample shape and the correlation between the directions of the current density $\bar{J}$ and the magnetic field $\bar{B}$.

In the case of cubic crystals (groups $\mathrm{m} 3 \mathrm{~m}, 432$ and $\overline{4} 3 \mathrm{~m})$ the problem is simplified because relation (1) can be expressed by the Pearson-Suhl (PS) expression proposed by J. Bardeen [6]

$$
\bar{E}=\rho_{0}\left[\bar{J}+\alpha \bar{J} \times \bar{B}+b B^{2} \bar{J}+c(\bar{J} \cdot \bar{B}) \bar{B}+\mathrm{d} T \bar{J}\right]
$$

where the PS coefficients $\alpha, b, c, d$ are defined by the following expressions

and

$$
\alpha=\frac{\rho_{123}}{\rho_{0}}, \quad b=\frac{\rho_{1122}}{\rho_{0}}, \quad c=\frac{2 \rho_{1212}}{\rho_{0}}
$$

$$
d=\frac{\rho_{1111}-\rho_{1122}-2 \rho_{1212}}{\rho_{0}} .
$$

Thus the determination of the $\rho_{i j k l}$ is reduced to the measurement of the $b, c, d$ coefficients.

Introducing the magnetoresistance coefficients

$$
\gamma_{\alpha \beta \gamma}^{\delta \varepsilon \zeta}=\frac{\Delta \rho}{\rho_{0} B^{2}}
$$

or the dimensionless ones [7]

$$
M_{\alpha \beta \gamma}^{\delta \varepsilon \zeta}=\frac{\Delta \rho}{\rho_{0}\left(\mu_{\mathrm{H}} B\right)^{2}}
$$

where $\mu_{\mathrm{H}}$ is the Hall mobility, the $b, c, d$ coefficients can be determined by selecting the proper directions $\alpha \beta \gamma$ of $\bar{J}$ and $\delta \varepsilon \zeta$ of $\bar{B}$.

So with the sample rod along the direction [100] only two combinations of $b, c, d$ are obtainable from $M_{100}^{100}$ and $M_{100}^{010}$ while with the sample rod along the direction [110] we have three combinations of $b, c, d$ from $M_{110}^{110}, M_{110}^{110}$ and $M_{110}^{001}$ which allow the determination of the three $\rho_{i j k l}$ coefficients.

Li et al. [8] working on the magnetoresistance of $\mathrm{Mg}_{2} \mathrm{Ge}$ succeed to determine the $b, c, d$ coefficients with $\bar{B}$ lying in a single plane - the $(\overline{1} 10)$ plane using a sample along the [112] direction. With this configuration the magnetoresistance coefficient $M_{112}^{\theta}$ (where $\theta$ is the angle between $\bar{B}$ and $\bar{J}$ ) is given by the equation

$$
\begin{aligned}
M_{1 \mathrm{i} 2}^{\theta}= & \frac{1}{12}(12 b+6 c+5 d)+ \\
& +\frac{1}{12}(6 c+d) \cos 2 \theta+\frac{d}{3 \sqrt{2}} \sin 2 \theta .
\end{aligned}
$$

An important result of this equation is that the extrema of the magnetoresistance in the direction [112] do not occur for $\theta=0^{\circ}$ or $\theta=90^{\circ}$. This new feature was called by Allgaier et al. [9-11] magnetoresistance skewness.

We may say that in cubic crystals only three diffe- rent WFMR measurements are possible on a single sample, because a fourth measurement is impossible or unnecessary with the classical sample shape of the orthogonal rod.

As the symmetry of the material is lowered, expression (1) can no longer be reduced to a simple PStype formula because the number of distinct nonzero coefficients increases considerably, and because the zero-field resistivity is, in general, no longer isotropic. In this case more than one sample and special configurations [12-14] are needed for the determination of all the WFMR coefficients.

Recently Allgaier et al. [9-11] have developed an extention of the PS formula applicable to (001) and (111) oriented layers and films, which is useful in distinguishing cubic from non cubic environments. The main characteristic concerning this extention is that the sample current lies along a nonspecial direction on the plane. Thus four distinct WFMR measurements may always be made on a single sample. From the phenomenology of this extention the skewness effect - as it is defined by Allgaier $e t$ al. - is predicted. But its actual physical meaning was not unveiled.

An application with good results of this four parameter extention has been made on (111) oriented epitaxial films of $\mathrm{n}$-type $\mathrm{PbTe}$ by Allgaier et al. [15].

In all the above cases the current is in some particular direction and the behaviour of the magnetoresistance is examined in the same direction, as the direction of the magnetic field is rotated in certain planes. Therefore with measurements of this kind we don't have the actual distribution of the magnetoresistivity on the sample plane when the magnetic field $\bar{B}$ is directed along an arbitrary direction.

\section{An alternative method for electric measurements.} - In 1958 van der Pauw introduced a method for measuring the GMC [16]. This method is based upon conformal mapping and it can be applied to isotropic materials and flat samples of arbitrary shape. About ten years later the method was extended for anisotropic materials by Wasscher [5] who solved the case of circular or rectangular samples. The use of a circular sample is more convenient because only its thickness is needed to be known. The main result in this extention is the determination of the main ratio of the in-plane resistivity anisotropy. Thus the direction and the values of the in-plane principal resistivities are obtained [17]. Also the method is suitable for measurements under the influence of the magnetic field.

Thus the distribution of the resistivity in the sample . plane is obtained using the well known formula [18]

$$
\rho(0)=\frac{\bar{E} \cdot \bar{J}}{J^{2}}=\rho_{i i} c_{i}^{2}
$$

where $\rho_{i i}$ are the principal resistivities and $c_{i}$ are the direction cosines of the current density $\bar{J}$. In the pre- 
sence of the magnetic field we have for the resistivity the expression

$$
\rho(\bar{B})=\frac{\bar{E}(\bar{B}) \cdot \bar{J}}{J^{2}}=\rho_{i j}(\bar{B}) c_{i} c_{j}
$$

As it is evident from equation (8) the magnetoresistivity $\rho(\bar{B})$ is connected directly with the WFMR coefficients $\rho_{i j k l}$ by means of the equation (1). Therefore selecting the appropriate direction of the magnetic field $\bar{B}$ we can determine the $\rho_{i j k l}$ coefficients in a direct way which does not depend on the crystal class of the material under study.

The advantage of the method is that the current density $\bar{J}$ does not need to be in any particular direction, therefore the number of the distinct $\rho_{i j k l}$ coefficients which can be determined from one sample is increased. The complete procedure for the measurements has been described previously (see for example the orthorhombic case [1]). Also the method can be used in the case of surface layers or epitaxial films in order to distinguish cubic from non-cubic environments [2].

The coefficient of the type $\rho_{i i i i}$ is determined with $\bar{B}$ parallel to the principal direction $\left[x_{i}\right]$ by measuring the change in the resistivity in this direction, while the coefficient of the type $\rho_{i i j j}$ is determined with

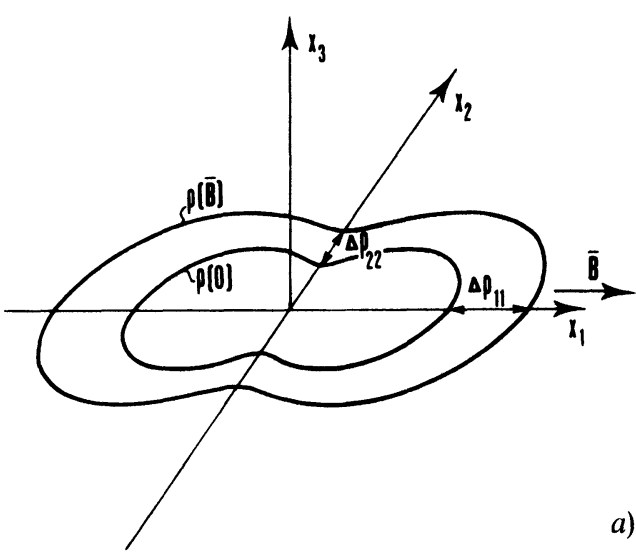

a)

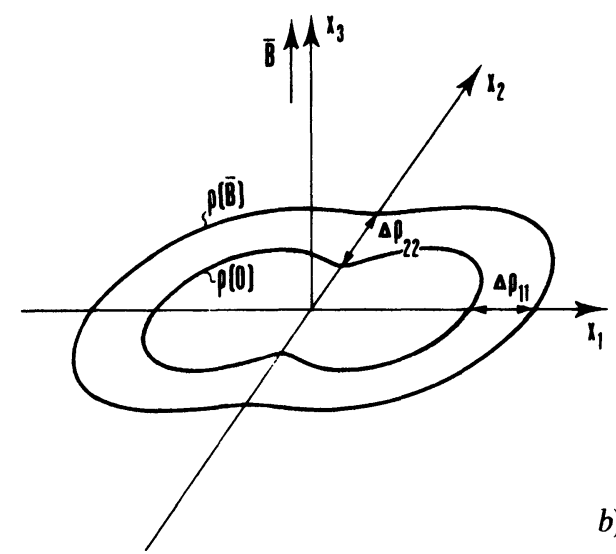

b)

Fig. 1. - The polar plott of resistivity for two cases : a) $\bar{B} / /\left[x_{1}\right]$ and $\left.b\right) \bar{B} / /\left[x_{3}\right]$. The measurement of $\Delta \rho_{i i}$ in the principal directions led to the determination of the corresponding $\rho_{i i j j}$ coefficient.
$\bar{B}$ directed along the $\left[x_{j}\right]$ direction, by measuring again the change in the resistivity in the direction $\left[x_{i}\right]$. In the first case we have the longitudinal magnetoresistance while in the second case the transverse one [19]. For example consider the profiles of the resistance anisotropy presented in figure 1 . From figure $1 a$ the coefficients

$$
\rho_{1111}=\frac{\Delta \rho_{11}}{B^{2}} \text { and } \rho_{2211}=\frac{\Delta \rho_{22}}{B^{2}}
$$

are determined, while from figure $1 b$ we can determine the coefficients

$$
\rho_{1133}=\frac{\Delta \rho_{11}}{B^{2}} \text { and } \rho_{2233}=\frac{\Delta \rho_{22}}{B^{2}} .
$$

The type $\rho_{i j i j}$ of the WFMR coefficients is determined by means of the MRS effect presented in the next paragraph.

4. The magnetoresistance skewness effect. - The skewness phenomenon is a consequence of the phenomenological theory of the weak-field case $[1,2]$. The application of the magnetic field introduces an electric anisotropy in the resistivity. This anisotropy has principal directions, in general, different from the directions of the original frame of reference which define the principal directions of the zero-field resistivity. Indeed from equation (8) it is evident that if there are elements $\rho_{i j}(\bar{B}) \neq 0$ for $i \neq j$ the tensor $\left[\rho_{i j}(\bar{B})\right]$ is not diagonal in the original system. We define as magnetoresistance skewness the change of the principal directions of the resistivity anisotropy due to the presence of the magnetic field.

The MRS with $\bar{B}$ lying in a definite direction on the sample plane e.g. the $\left(x_{1} x_{2}\right)$ plane is presented in figure 2. The angle of skewness $\varphi$ is the angle formed between the axes $x_{i}$ and $x_{i}^{\prime}$. In this case it can be proved [4] that the following expressions hold in general

$$
\begin{aligned}
\tan 2 \varphi & =\frac{2 \rho_{12}(\bar{B})}{\rho_{11}(\bar{B})-\rho_{22}(\bar{B})} \\
\rho_{11}^{\prime}(\bar{B}) & =\frac{1}{2}\left[\rho_{11}(\bar{B})+\rho_{22}(\bar{B})\right]+\frac{\rho_{12}(\bar{B})}{\sin 2 \varphi} \\
\rho_{22}^{\prime}(\bar{B}) & =\frac{1}{2}\left[\rho_{11}(\bar{B})+\rho_{22}(\bar{B})\right]-\frac{\rho_{12}(\bar{B})}{\sin 2 \varphi}
\end{aligned}
$$

where the primed quantities are refered to the new axes of the anisotropy. In such a case the tensor element $\rho_{12}(\bar{B})$ depends usually on the $\rho_{1212}$ coefficient and the direction of $\bar{B}$. If the direction cosines of $\bar{B}$ are $u, v, 0$ in respect to the $x_{i}$ axes we have the relation [1-4]

$$
4 \rho_{1212} B^{2} u v=\left[\rho_{11}^{\prime}(\bar{B})-\rho_{22}^{\prime}(\bar{B})\right] \sin 2 \varphi
$$

which is used for the experimental determination of the $\rho_{1212}$ coefficient. 


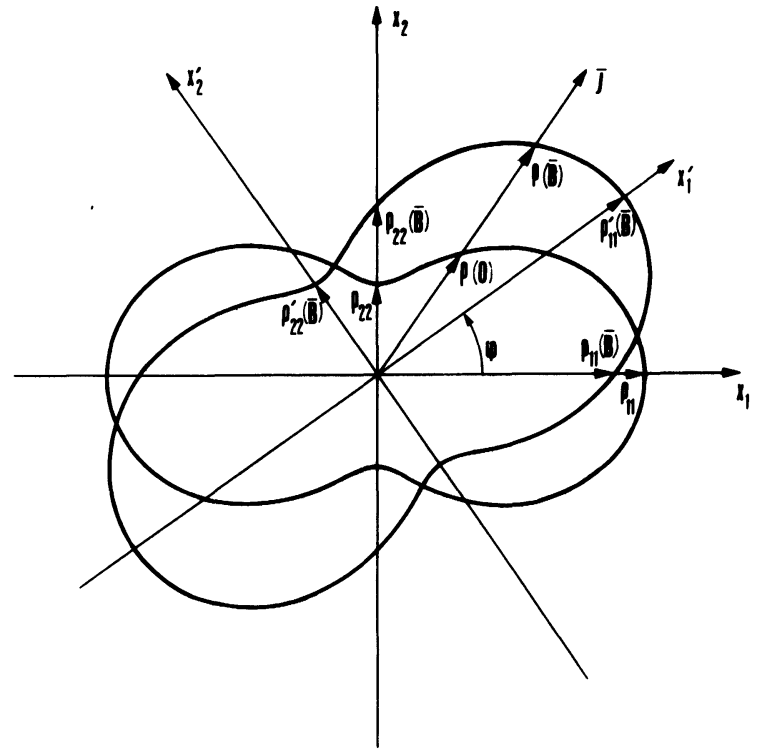

Fig. 2. - The presence of the magnetic field induces an anisotropy in the resistivity with principal directions different from the directions of the zero-field resistivity.

From equation (12) we may conclude that the $\rho_{i j i j}$ coefficients express the difference between the two principal magnetoresistances and the imposed skewness by the magnetic field. This definition has more physical meaning than the connection of the $\rho_{i j i j}$ coefficient with the planar Hall effect [19]. Such a measurement (eq. (12)) is attainable using Wasscher's method which allows the determination of the principal resistivities $\rho_{11}^{\prime}(\bar{B}), \rho_{22}^{\prime}(\bar{B})$ and that of the skewness angle $\varphi$. The experimental procedure is described in reference [4].

It should be noted that the determination of a coefficient of the type $\rho_{i i j k}$ or $\rho_{i i i j}$ is based again on the MRS effect with the direction of $\bar{B}$ inclined to the sample plane.

5. Results on n-type Ge. - Since the main point of the work in reference [4] is the determination of the magnitude of the GMC, and not merely the symmetry of the system as in previous works in this field, we think that it would be interesting to explore the information obtained concerning the physical significance of the coefficients measured and the material parameters that may be determined from the measurements.

All the measurements were made at room temperature on the (001)-plane of n-type Ge using the above described method. The experimental values of the GMC are given in the table I. Previous measurements on n-Ge [6, 22-24] have shown that the PS coefficients must satisfy the following conditions

$$
d>0 \text { and } b+c=0 .
$$

The two conditions imply that the $\rho_{1111}$ coefficient has a non zero value and so the material exhibit longitu-
Table I. - The galvanomagnetic coefficients of the n-type Ge sample (SI units)

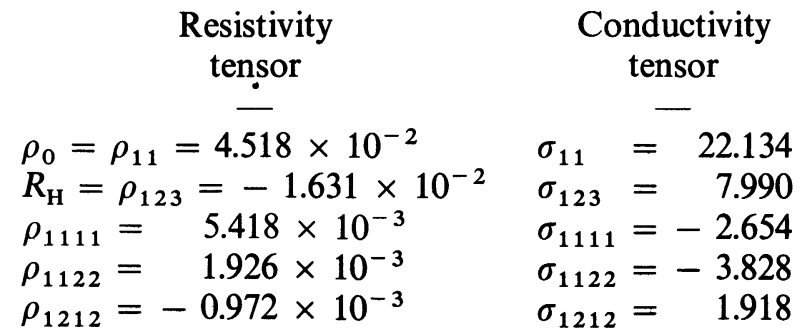

dinal magnetoresistance, while the second condition shows that the coefficients $b$ and $c$ have opposite signs. In fact the coefficient $c$ has a negative value which means that the corresponding WFMR coefficient $\rho_{1212}$ is also negative. The two other coefficients $\rho_{1111}$ and $\rho_{1122}$ have positive values with $\rho_{1111}>\rho_{1122}$.

Therefore the appropriate energy band model for $\mathrm{n}-\mathrm{Ge}$ consists of a set of ellipsoids of revolution oriented along the [111] directions of the reciprocal space $[20,21]$. If the elements of the effective-mass tensor are $m_{1}=m_{2}=m_{1}$ and $m_{3}=m_{1}$, the resistivity and conductivity tensors can be written in terms of the effective mass ratio $K=m_{1} / m_{\mathrm{t}}$ and of the relaxation time $\tau$. The expressions for the resistivity and conductivity tensors and the relations between the elements of them are well known (see for example references [14] and [19]). So it was considered that the negative sign of the $\rho_{1212}$ coefficient is a consequence of the energy band model of the material only, without any other physical meaning.

Equation (9) in the case of the (001)-plane takes the form $[3,4]$

$$
\tan 2 \varphi=\frac{4 \rho_{1212} u v}{\left(\rho_{1111}-\rho_{1122}\right)\left(u^{2}-v^{2}\right)}
$$

which means that the angle of skewness depends only on the direction of $\bar{B}$ and the relative values of the three WFMR coefficients. When $\rho_{1111}>\rho_{1122}$, as it is in this case, the $\rho_{1212}$ coefficient has a negative values if the angle of skewness $\varphi$ is also negative. This means that the main directions of magnetoresistivity must rotate in a reverse course with respect to the rotation of the direction of the magnetic field. Indeed, as it is referred in [4], a reverse rotation of the magnetoresistance was observed in $\mathrm{n}-\mathrm{Ge}$. This situation is presented in figure 3 where the polar distribution of the zero field resistivity and of the magnetoresistivity are drawn for a definite direction of the magnetic field $\bar{B}$. As we see from this figure $\rho_{11}^{\prime}(\bar{B})>\rho_{22}^{\prime}(\bar{B})$ and since the angle of skewness is negative, from equation (12) it follows that the $\rho_{1212}$ coefficient has a negative value.

Therefore we may conclude that for n-type Ge the negative value of the $\rho_{1212}$ coefficient is connected with the reverse rotation of the magnetoresistance and this is the actual physical meaning of the negative 


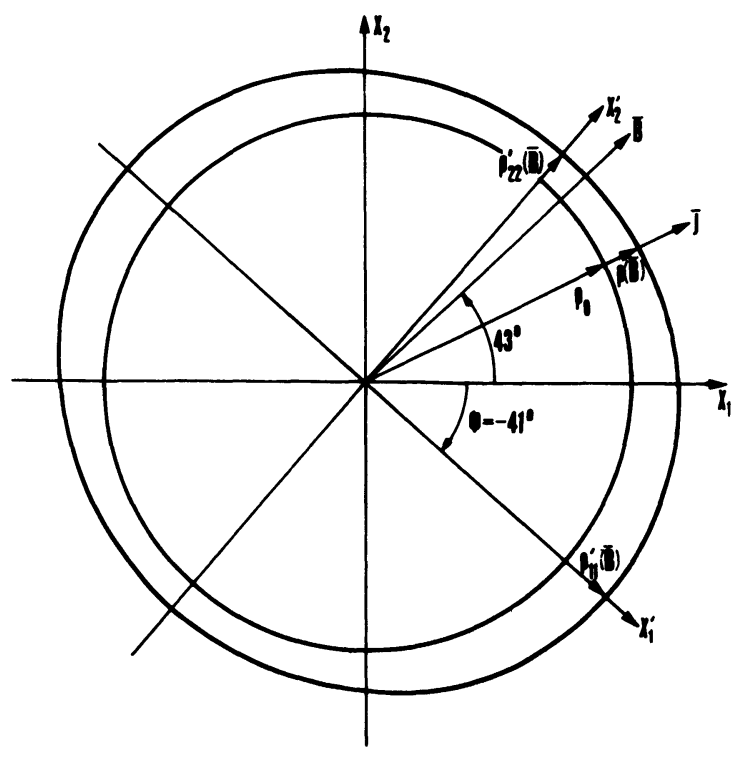

Fig. 3. - Magnetoresistance skewness effect on the (001plane of n-Ge. The directions $\left[x_{1}\right]$ and $\left[x_{2}\right]$ are the crystallographic directions [100] and [010] respectively [4].

sign. This fact reinforces the previous definition that the $\rho_{1212}$ coefficient expresses the imposed skewness by the magnetic field.

Another feature of the $\langle 111\rangle$ multivalley model is the constant value of the ratio $\rho_{1212} / \rho_{1122}$ (or $\left.\sigma_{1212} / \sigma_{1122}\right)$. From table I it follows that the experimental value of the ratio $\rho_{1212} / \rho_{1122}$ is $-0.505 v s$. -0.500 which is predicted from the energy model.

For this model the ratio $K$ is determined by the ratio $\sigma_{1111} / \sigma_{1122}$. So we have two values, $K_{\mathrm{p}}=12.090$ for the prolate spheroid and $K_{0}=0.083$ for the oblate one. It is remarkable that these two values are reversal, so $K_{\mathrm{p}} K_{0}=1$. The explanation lies in the fact that the ratio $\sigma_{1111} / \sigma_{1122}$ is expressed by the same function of $K=m_{1} / m_{\mathrm{t}}$ or of $K^{\prime}=m_{\mathrm{t}} / m_{1}$. However the same is true also in the case of the six-valley model in [100] directions as deduced from the measurements of Li et al. [8] on $\mathrm{Mg}_{2} \mathrm{Ge}$ (Table III of ref. [8]).

The value $K_{\mathrm{p}} \simeq 12$ is in agreement with previous measurements [22-24] and actually expresses the ratio

$$
\frac{K_{\mathrm{m}}}{K_{\tau}}=\frac{m_{1} \tau_{\mathrm{t}}}{m_{\mathrm{t}} \tau_{1}}
$$

and not only the effective-mass ratio [25].

Now, the scattering factor $r=\langle\tau\rangle\left\langle\tau^{3}\right\rangle\left\langle\left\langle\tau^{2}\right\rangle^{2}\right.$ may be found from the ratio $\rho_{1111} / \rho_{1122}$. According to the Schwartz inequality it is always $r \geqslant 1$. So using the above value of $K_{\mathrm{p}}$ we find

$$
r=1.070 \text {. }
$$

Assuming that the relaxation time is a function of the energy only in the form

$$
\tau=\tau_{0} \varepsilon^{q}
$$

the scattering factor takes the usual expression

$$
r=\frac{\Gamma\left(\frac{5}{2}+3 q\right) \Gamma\left(\frac{5}{2}+q\right)}{\left[\Gamma\left(\frac{5}{2}+2 q\right)\right]^{2}}
$$

For scattering by lattice vibrations the theoretical value of $q$ is -0.5 which leads to a value $r=1.272$. Measurements on pure $\mathrm{n}-\mathrm{Ge}[23,26,27]$ gave a value $q \simeq 0.65$ and so $r=1.813$. Using for $r$ the value from (16) we find that the exponent in equation (17) has a value $q=-0.31$. The deviation from the value -0.65 shows that ionized impurities are important as scattering centers, as it has been suggested by previous workers [23, 24, 28, 29].

The concentration of the charge carriers is determined by the relation

$$
n=-\frac{1}{R_{\mathrm{H}} e} \frac{3 K(K+2)}{(1+2 K)^{2}} \frac{\left\langle\tau^{2}\right\rangle}{\langle\tau\rangle^{2}} .
$$

Using the values $K=12.090$ and $q=-0.31$ we find

$$
n=3.265 \times 10^{20} \mathrm{~m}^{-3}=3.265 \times 10^{14} \mathrm{~cm}^{-3} \text {. }
$$

The drift mobility is equal to

$\mu_{\mathrm{D}}=\frac{\sigma}{n e}=0.424 \mathrm{~m}^{2} \mathrm{~V}^{-1} \mathrm{~s}^{-1}=4240 \mathrm{~cm}^{2} \mathrm{~V}^{-1} \mathrm{~s}^{-1}$

while the Hall mobility is

$\mu_{\mathrm{H}}=R_{\mathrm{H}} \sigma=0.361 \mathrm{~m}^{2} \mathrm{~V}^{-1} \mathrm{~s}^{-1}=3610 \mathrm{~cm}^{2} \mathrm{~V}^{-1} \mathrm{~s}^{-1}$.

Now the ratio $\mu_{\mathrm{H}} / \mu_{\mathrm{D}}$ is found to be

$$
\frac{\mu_{\mathrm{H}}}{\mu_{\mathrm{D}}}=R_{\mathrm{H}} n e=\frac{3 K(K+2)}{(1+2 K)^{2}} \frac{\left\langle\tau^{2}\right\rangle}{\langle\tau\rangle^{2}}=0.852 .
$$

As we see the Hall mobility is less than the drift mobility and the value of the ratio $\mu_{\mathrm{H}} / \mu_{\mathrm{D}}$ depends on the values of $K$ and $q$, e.g. on the special band model and the scattering mechanism. Therefore even in the case of pure $\mathrm{Ge}$, where $q=-0.65$, it is $\mu_{\mathrm{H}}<\mu_{\mathrm{D}}$, assuming that $K=12$.

6. Comparison with the classical configuration. Although the previous results fit perfectly with the in common use energy band model for the n-Ge, we have made additional measurements at room temperature using the classical configuration described in section 2 .

The sample rod was cut along the [110] direction from the same bulk crystal as the sample used in reference [4]. For the magnetoresistance measurements we have used two configurations for the magnetic field $\bar{B}$. In the first the field $\bar{B}$ was rotated in the (001)-plane and in the second in a vertical plane formed by the directions [001] and [110]. In figure 4 we show the results of the magnetoresistance measurements. From 


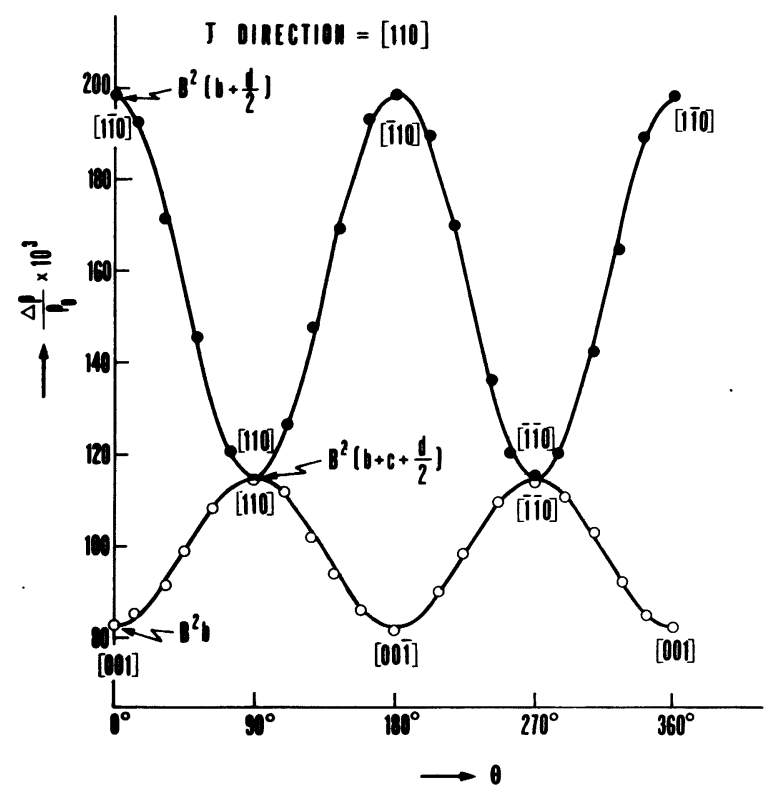

Fig. 4. - Variations of $\Delta \rho / \rho_{0}$ in the [110] sample rod as $\bar{B}$ is rotated through an angle $\theta$ from 0 to 360 degrees in two vertical planes. The points which are used for the determination of the $b, c, d$ coefficients are indicated.

this figure is evident that the extrema occur at angles $0^{\circ}$ or $90^{\circ}$ and of course a skewed behaviour does not exist. Also in the same figure we have indicated the points from which we can determine the $b, c, d$ coefficients. Using equation (3) we obtain the values of the $\rho_{i j k l}$ coefficients. In table II we give the results of these measurements. As we see the conditions (13) for the $b$, $c, d$ coefficients are valid while the values of the GMC are in a good agreement with the values of the table I. The comparison shows that in fact we have the same
Table II. - The results from the measurements on the [110] sample rod with $B=1.4 \mathrm{~T}$ (SI units)

$$
\begin{aligned}
& b=42.001 \times 10^{-3} \quad \rho_{0}=4.488 \times 10^{-2} \\
& c=-42.692 \times 10^{-3} \quad \rho_{1111}=5.295 \times 10^{-3} \\
& d=118.672 \times 10^{-3} \quad \rho_{1122}=1.885 \times 10^{-3} \\
& \rho_{1212}=-0.958 \times 10^{-3}
\end{aligned}
$$

numerical results but the information which we have for the behaviour of the material is less compared with that obtainedby the method described in sections 3 and 4.

7. Conclusions. - This work concerns the experimental methodology for the electrical measurements mainly those of the magnetoresistance. The comparison is made between the traditional method and the relatively new Wasscher's method. For the experiments we have used a classical semiconductor the n-Ge which is a very well studied material. The main advantage of the Wasscher's method is that the current density $\bar{J}$ is not in a fixed direction on the sample plane but corresponds to a rotation in it. Therefore the information which we have about the macroscopic behaviour of the material and the physical significance of the measured coefficients are more complete and their significance easily revealed. For example the MRS effect which is very intense for the cubic crystals was unknown and no physical meaning could be found for the negative sign of the $\rho_{1212}$ coefficient of n-Ge. On the other hand the determination of the magnetoresistance coefficients is immediate and it is not dependent from the crystal symmetry. Finally the comparison with limited classical configurations confirm the reliability of the methodology advanced here.

References

[1] Kyriakos, D. S. and Economou, N. A., Phys. Status Solidi (b) 94 (1979) 549.

[2] Kyriakos, D. S., Economou, N. A. and Allgaier, R. S., Revue Phys. Appl. 15 (1980) 733.

[3] Kyriakos, D. S. and Economou, N. A., Appl. Phys. Lett. 35 (1979) 894.

[4] Kyriakos, D. S., Valassiades, O. E., PaPadimiTriou, K. G. and Economou, N. A., J. Appl. Phys. (In press).

[5] Wasscher, J. D., Phil. Res. Rep., Suppl. 8 (1969) 1.

[6] Pearson, G. L. and Suht, H., Phys. Rev. 83 (1951).

[7] Allgaier, R. S., Phys. Rev. 115 (1959) 1185.

[8] Li, P. W., Lee, S. N. and Danielson, G. C., Phys. Rev. B 6 (1972) 442.

[9] Allgaier, R. S. and Restorff, J. B., J. Appl. Phys. 50 (1979) 402.

[10] Allgaier, R. S., Restorff, J. B. and Bland HousTON, Phys. Rev. B 19 (1979) 6155.

[11] Allgaier, R. S., Restorff, J. B. and Bland Houston, Appl. Phys. Lett. 34 (1979) 158.

[12] Drabble, J. R., Groves, R. D. and WolfF, R., Proc. Phys. Soc. 71 (1958) 430.

[13] Drabble, J. R., Proc. Phys. Soc. 72 (1958) 380.

[14] BEER, A. C., Galvanomagnetic Effects in Semiconductors (Academic Press, London) 1963.
[15] Allgaier, R. S., Restorff, J. B., Bland Houston, Jensen, J. D. and Lopez-Otero, A., J. Appl. Phys. 51 (1980) 2119.

[16] Van der Pauw, L. J., Phil. Res. Rep. 13 (1958) 1.

[17] Kyriakos, D. S. and Economou, N. A., Sci. Annals., Fac. Phys. and Mathem. (Univ. Thessaloniki) 19 (1979) 175.

[18] NyE, J. F., Physical properties of Crystals (Oxford Univ. Press, London) 1967.

[19] Smith, A. C., JANAK, J. F. and AdLer, R. B., Electronic Conduction in Solids (McGraw-Hill, New York) 1967.

[20] Abeles, B. and-Meiboon, S., Phys. Rev. 95 (1954) 31.

[21] Shibuya, M., Phys. Rev. 95 (1954) 1385.

[22] Glicksman, M., Phys. Rev. 100 (1955) 1146.

[23] Goldberg, C. and Davis, R. E., Phys. Rev. 102 (1956) 1254.

[24] Glicksman, M., Phys. Rev. 108 (1957) 264.

[25] Herring, C. and Vogt, E., Phys. Rev. 101 (1956) 944.

[26] Prince, M. B., Phys. Rev. 92 (1953) 681.

[27] Morin, F. J., Phys. Rev. 93 (1954) 62.

[28] Benedek, G. B., Paul, W. and Brooks, H., Phys. Rev. 100 (1955) 1129.

[29] Ham, F. S., Phys. Rev. 100 (1955) 1251 (A). 